\title{
Single crystal monolithic up-converter solar cell device tandems with integrated optics
}

Georgios E. Arnaoutakis ${ }^{1^{*}}$, Elena Favilla ${ }^{2}$, Mauro Tonelli ${ }^{2,3}$ and Bryce S. Richards ${ }^{4,5 *}$

1 Department of Mechanical Engineering, Hellenic Mediterranean University, Estavromenos, Heraklion Crete 710 04, Greece

${ }^{2}$ Dipartimento di Fisica - Universita' di Pisa, Largo B. Pontecorvo, 3 I-56127 PISA, Italy

${ }^{3}$ Megamaterials srl Largo B. Pontecorvo , 356127 I Pisa

${ }^{4}$ Institute of Microstructure Technology (IMT), Karlsruhe Institute of Technology, Hermann-vonHelmholtz-Platz 1, 76344 Eggenstein-Leopoldshafen, Germany

${ }^{5}$ Light Technology Institute (LTI), Karlsruhe Institute of Technology, Engesserstrasse 13, 76131 Karlsruhe, Germany

*Corresponding author: E-mail: bryce.richards@kit.edu (Prof.Bryce Richards), arnaoutakis@hmu.gr (Dr Georgios Arnaoutakis)

\begin{abstract}
Solar photons possessing energy less than the band-gap of a single-junction solar cell can be utilized via the up-conversion (UC) of two or more photons, resulting in the emission of a single abovebandgap photon. Due to the non-linear nature of $\mathrm{UC}$, highly concentrated light is required, which is typically much greater than the practical concentration limits of a solar cell. It has been proposed that concentrating up-conversion solar cells (UC-SC) with optical elements integrated into the device could help realize the high solar irradiance required. To avoid scattering problems arising from common UC materials based on micro-crystalline powders, in this work concentrators are investigated with monocrystalline up-converters in silicon-based tandem devices. An external quantum efficiency (EQE) of $6 \%$ with $1493 \mathrm{~nm}$ infrared illumination at $876 \mathrm{~W} / \mathrm{m}^{2}$ was obtained in upconverter device with concave integrated optics. At an irradiance higher than $90 \mathrm{~W} / \mathrm{m}^{2}$ (equivalent to $2.95 \times$ in the $1450-1600 \mathrm{~nm}$ range), the non-concentrating UC-SC exhibited 1.5× higher EQE than the UC-SC with CPC, while below $90 \mathrm{~W} / \mathrm{m}^{2}$ the CPC UC-SC exhibited 1.95× higher EQE than the non-concentrating reference device. Due to the negligible scattering of the UC layer, the distribution of localized irradiance is revealed along with its effect on the performance of devices. It is found that irradiance is accumulated within the first $1 \mathrm{~mm}$ of the UC layer with peaks at variable depths according to the concentrating scheme. These results suggest ample space for improved up-conversion devices by using integrated optics.
\end{abstract}

Keywords: Photovoltaics, Up-conversion, Erbium, Silicon, Compound Parabolic Concentrator, integrated optics.

\section{Introduction}

Up-conversion (UC) is one of the emerging third generation photovoltaic (PV) approaches to improve the sub-bandgap response of single-junction solar cells. It involves the conversion of two or more low energy photons into one photon possessing energy greater than the bandgap. For crystalline silicon (c$\mathrm{Si}$ ) solar cells with an (indirect) bandgap of $E_{g}=1.12 \mathrm{eV}$, such near infrared (NIR) transparency losses account for more than $20 \%$ of the energy in the solar spectrum [1], and capturing this could improve the conversion efficiency of a solar cell by $20 \%$ [2,3]. To date, the majority of UC research applied to solar energy harvesting has been oriented towards c-Si solar cells as this technology represents $>94 \%$ of today's PV production globally [4]. The vast majority of c-Si PV modules are designed to operate under one-sun illumination $\left(1000 \mathrm{~W} / \mathrm{m}^{2}\right)$ conditions. There exist some exceptions where c-Si solar cells operated at solar concentrations ranging from 10 suns $\left(10,000 \mathrm{~W} / \mathrm{m}^{2}\right)$ [5], to 40 suns [6] up to 92 suns $[7,8]$. Solar cells made from c-Si are not suited for operation at higher solar concentration due to series and contact resistance [9], but more important Auger recombination beginning to dominate and significantly reducing the conversion efficiency [10]. This latter fact is unfortunate as UC is a multiphoton non-linear process and thus the required solar concentration on the up-converter can be more 
than an order of magnitude higher [11-13]. A recent review by Richards et al. indicated that at least a 100-times increase in the incident photon flux (or alternatively the intermediate state lifetime of the UC mechanism) is required to start seeing efficient solar UC, noting that, even then, many assumptions were very optimistic [14]. Trivalent erbium $\left(\mathrm{Er}^{3+}\right)$-doped up-converters are suitable for silicon solar cell tandems, due to their efficient up-conversion of $\sim 1500 \mathrm{~nm}$ light to $\sim 980 \mathrm{~nm}$ emission, which lies just above the bandgap of c-Si. Such up-converters exhibit an increased quantum yield at higher than 350 suns [12] with an onset of saturation at 100,000 suns [15], although noting the level of saturation is dependent on other factors such as $\mathrm{Er}^{3+}$ ion concentration [16] and it has been recently reported that thermal effects definitely need to be considered at high concentration [17]. Broadband upconverters in the $1500-1750 \mathrm{~nm}$ as well as the $1100-1400 \mathrm{~nm}$ band would be promising for a greater utilization of the solar spectrum. In addition to the approach of sunlight concentration presented in the current work, there are a number of routes for enhancing upconverters discussed in detail in [14]. A broader spectrum can be achieved by co-doping $\mathrm{Er}^{3+}$ with additional lanthanide ions. $\mathrm{Dy}^{3+}, \mathrm{Yb}^{3+}$ and $\mathrm{Ho}^{3+}$ have been reported as sensitizers. Transition metals such as $\mathrm{Ni}^{2+}$ exhibit wide absorption in 1100-1400 $\mathrm{nm}$. A high energy transfer efficiency to $\mathrm{Er}^{3+}$ was recently reported in $\mathrm{Ni}^{2+}$-, $\mathrm{Er}^{3+}$-doped ceramics [18]. Quantum dots were also reported to shift the $1100-1400 \mathrm{~nm}$ band [19]. Both reports focused on energy transfer to the first excited level of erbium, despite several metastable levels exist in this band. Unfortunately, the limited quantification of the absolute upconversion quantum yield (PLQY) does not permit an evaluation of the practical gains for a solar cell. Two of the most efficient upconverters reported to-date are $\mathrm{BaY}_{2} \mathrm{~F}_{8}: 30 \% \mathrm{Er}^{3+}$ with internal $\mathrm{UCQY}=10.1 \%$ and $\beta-\mathrm{NaYF}_{4}: 25 \% \mathrm{Er}^{3+}$ with $\mathrm{PLQY}=12 \%$ at $0.4 \mathrm{~W} / \mathrm{cm}^{2}$. These upconverters resulted in $8 \%$ and $4.4 \%$ solar cell EQE at $0.4 \mathrm{~W} / \mathrm{cm}^{2}$ infrared illumination, respectively [20]. The EQE of organic upconverters approaches $30 \%$ as summarized by Pedrini and Monguzzi [21]. However, the conversion is limited in the 340-1010 $\mathrm{nm}$ range, therefore not yet suitable for silicon solar cells.

In a previous work, the present authors demonstrated that a higher concentration of light on the upconverter can be achieved via the inclusion of optical elements between the bifacial c-Si solar cell and the up-converter [22]. Furthermore, an efficiency enhancement equal to the concentration ratio of $2.8 \times$ has been reported by using compound parabolic concentrators (CPC) [23]. This use of optics enabled the optimization of the solar concentration independently from the overlying solar cell [24,25]. Such results were demonstrated using a micro-crystalline powder of hexagonal erbium-doped sodium yttrium fluoride $\left(\beta\right.$-NaYF: $\left.25 \% \mathrm{Er}^{3+}\right)$, one of the best performing up-converters for c-Si solar cells todate [20]. For an extensive recent review of up-conversion materials, solar cells and devices, the interested reader is directed towards reference [20] as well as the more recent critical review of UC for PV and photocatalysis, based on photophysical analysis [21]. It is important to clarify that another micro-crystalline up-converter, gadolinium oxysulfide $\left(\mathrm{Gd}_{2} \mathrm{O}_{2} \mathrm{~S}: 10 \% \mathrm{Er}^{3+}\right)$ exhibited higher quantum yield under monochromatic excitation. However, under broadband excitation, more relevant to the application in solar cells, $\beta-\mathrm{NaYF}_{4}: 25 \% \mathrm{Er}^{3+}$ exhibited a higher quantum yield [26], and further demonstrated external quantum efficiency (EQE) of $1.79 \%$ at $1000 \mathrm{~W} / \mathrm{m}^{2}$ [27] and a $\Delta \mathrm{Jsc}$ of $9.4 \mathrm{~mA} / \mathrm{cm}^{2}$ due to the up-converter at 94 suns [28] with c-Si solar cells.

In this work, we seek to move away from the use of micro-crystalline powders. With a crystal size of $2-200 \mu \mathrm{m}$ [13], increased scattering inhibits the knowledge of light distribution inside the microcrystalline up-converters and its performance. A previous work by Arnaoutakis et al. [23] found an increase in $\mathrm{EQE}$ from $1.33 \%$ to $1.80 \%$ by the integration of CPC. In this paper, motivated by the high quantum yield, EQE and negligible scattering of erbium-doped barium yttrium fluoride $\left(\mathrm{BaY}_{2} \mathrm{~F}_{8}: 30 \% \mathrm{Er}^{3+}\right)$ single crystals $[29,30]$, we investigate the effect of the concentrators on monocrystalline up-converters and their performance on up-converter solar cell tandems.

\section{Materials and Methods}


The different configurations investigated within this work are shown in Figure 1(a-d). Reference devices (a) comprised of an up-converter layer (red) and a planar, bifacial c-Si solar cell (blue) were investigated together with different optical elements: (b) a concave reflector, (c) a CPC of acceptance angle $25^{\circ}$ and (d) a CPC of acceptance angle $45^{\circ}$. In (e) the main transitions leading to conversion of near $1500 \mathrm{~nm}$ light in the up-converter, are displayed in the energy level diagram. Excitation around $1493 \mathrm{~nm}$ will be absorbed via ground state absorption (GSA) to the ${ }^{4} I_{13 / 2}$ level. From this level, energy can be transferred and up-converted (ETU) resonantly between two nearby $\mathrm{Er}^{3+}$ ions. Depending on the population of each level, process ETU1 between ${ }^{4} I_{13 / 2}-{ }^{4} I_{9 / 2}$, ETU2 between ${ }^{4} I_{11 / 2}-{ }^{4} F_{9 / 2}$ or ETU3 between ${ }^{4} F_{9 / 2}-{ }^{4} F_{7 / 2}$ may occur. From the upper levels of each process, following several multi-phonon relaxations, emission to the ground state can occur resulting to light around $517 \mathrm{~nm}, 650 \mathrm{~nm}, 790 \mathrm{~nm}$ and $970 \mathrm{~nm}[30,31]$.
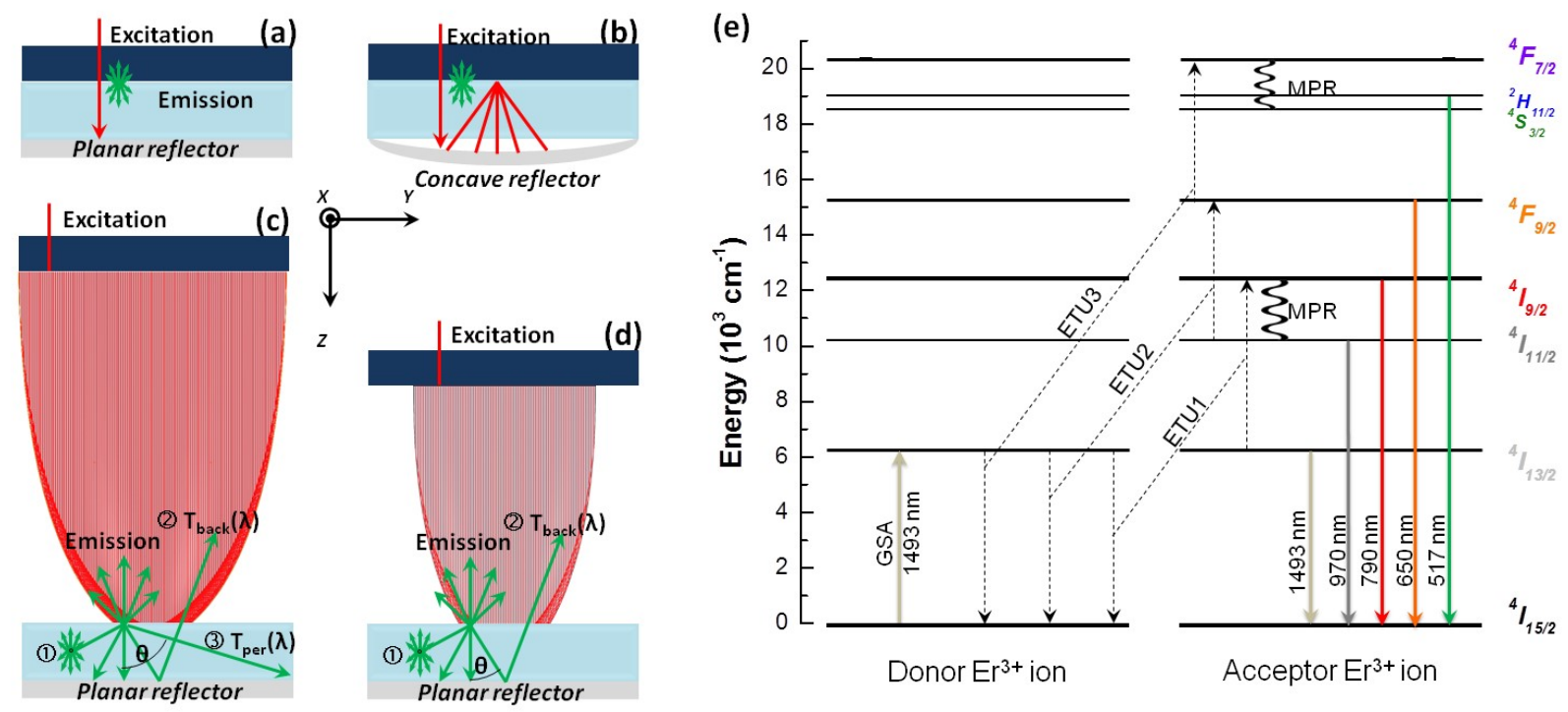

Figure 1: Four different configurations are investigated in this paper, with the up-converter shown in ruby and the bifacial cSi solar cell drawn in blue. In the reference scenarios, the excitation is at normal incidence and the emission is reflected by a specular planar back reflector. Note that no concentrating element is employed in the reference scenario. In (b) the concave reflector concentrates any non absorbed excitation at the up-converter solar cell interface. In (c) and (d), the introduction of the CPC-25 and CPC-45 elements respectively into the configuration results in NIR light entering the upconverter at oblique angles. The isotropic up-converted emission is externally coupled back into the CPC and delivered to the overlying bifacial c-Si solar cell. Internally, the emission may be (1) re-absorbed and re-emitted, (2) reflected at the back reflector and transmitted towards the solar cell, or (3) lost via the peripheral facets of the up-converter. (d) The energy level diagram depicts the main transitions taking place in the $\mathrm{Er}^{3+}$-doped up-converter with upward arrows indicating the absorption of light, downward arrows the resulting emission, (--) non-radiative energy transfer up-conversion (ETU) and ( ) multi-phonon relaxation (MPR).

\subsection{Simulation}

The geometrical loss mechanisms occurring in the studied configurations were calculated using ray optics and Monte Carlo simulations (Optis, Optisworks) in three-dimensional models using $>10^{7}$ rays over an area of $100 \mathrm{~mm}^{2}$. Non-sequential ray tracing was used with $0.5 \mathrm{~mm}$ facet width used for tesselation of each optical element. The simulation included the refractive indices and absorption coefficients of the CPC optics (Schott B270) and the $\mathrm{BaY}_{2} \mathrm{~F}_{8}: 30 \% \mathrm{Er}^{3+}$ material itself [31,32]. Empirical data [33] were used for the refractive index of Schott B270, while for $\mathrm{BaY}_{2} \mathrm{~F}_{8}$ coefficients were fit in a modified Sellmeier equation of the form [32]: 


$$
n^{2}=A+\frac{B \lambda^{2}}{\lambda^{2}-C}+\frac{D \lambda^{2}}{\lambda^{2}-E}
$$

, where $\lambda$ is the wavelength and the Sellmeier coefficients $A=1.04037, B=1.12565, C=0.00686$, $\mathrm{D}=0.2038$ and $\mathrm{E}=66.043$. The resultant fit is in good agreement with the dispersion of $\mathrm{BaY}_{2} \mathrm{~F}_{8}: 30 \% \mathrm{Er}^{3+}$ recently determined by high accuracy variable angle spectroscopic ellipsometry [34]. Further details on the simulation can be sourced in [35].

Near-infrared (NIR) excitation light at the peak absorption wavelength (1493 nm) and a Gaussian intensity profile was used with power density, $P_{\text {exc }}$, normally incident on the top surface of the devices (as depicted in Figure 1(a-d). Planar wave illumination accurately represents non-concentrated airmass 1.5 global (AM1.5G) sunlight, while Gaussian illumination better matches concentrated sunlight conditions. The rationale for using Gaussian illumination in the simulations was primarily driven by the assumption that a low concentration optical element is more likely to be used for primary concentration on top of silicon to reduce the concentration requirement of the secondary integrated optical element. Gaussian illumination also describes the distribution of lasers used for excitation, thereby more accurately simulating the conditions used in the presented experiments.

The NIR excitation was reduced by the transmittance of the up-conversion optimized c-Si solar cell, that is $85 \%$ at $1493 \mathrm{~nm}$, while the c-Si solar cell exhibits a rear-side reflectance of $8 \%$ for the UC light emitted between $980-1050 \mathrm{~nm}$ [36]. The emitted power, $P_{e m}$, was calculated as $P_{e m}(\lambda)=P_{e x c}(\lambda) \times i P L Q Y(\lambda)$ by considering the excitation power $P_{e x c}$, the internal photoluminescence quantum yield (iPLQY) of the UC material at the main radiative UC emission from state ${ }^{4} I_{11 / 2}$ to the ground state at $970 \mathrm{~nm}$. It is important to note that the iPLQY is defined as the ratio of emitted photons to the number of absorbed photons, in contrast to the external PLQY (ePLQY) which is defined as the ratio of emitted photons to the number of incident photons. Thus, the ePLQY is more relevant for realworld devices as the impact of optical losses, such as incomplete absorption, are included in this figureof-merit. The back reflectors were modeled as ideal specular. The wavelength dependent absorptance and path-length of reference and CPC cases were analytically calculated following the Beer-Lambert law as:

$$
A_{\text {Ref }}=1-e^{-\alpha 2 z}, A_{c o n}=1-e^{-\alpha 2 z / \cos \theta}
$$

, where $z=0.233 \mathrm{~cm}, \theta_{\text {conc }}=50^{\circ}, \theta_{C P C-25}=67^{\circ}, \theta_{C P C-45}=35^{\circ}$.

\subsection{Experiment}

Specular planar reflectors based on thin films of gold $(\mathrm{Au})$ of at at least $100 \mathrm{~nm}$ thickness were evaporated onto fused silica $\left(\mathrm{SiO}_{2}\right)$ slides. A silver-coated concave mirror with $\mathrm{f}=17.5 \mathrm{~mm}$, $\mathrm{R}=35 \mathrm{~mm}$ and average infrared reflectance higher than $95 \%$ was used in a $2 \mathrm{f}$ configuration replacing the planar back reflector. Two geometries of CPC fabricated from Schott B270 glass with acceptance angles $25^{\circ}$ and $45^{\circ}$ (Edmund Optics, USA) were utilised in the experiments. The dimensions for these were: entry aperture $\varnothing 9.01 \mathrm{~mm}$ and $\varnothing 5.39 \mathrm{~mm}$, and length $19.93 \mathrm{~mm}$ and $7.52 \mathrm{~mm}$, respectively, while the exit aperture for both was $\varnothing 2.5 \mathrm{~mm}$. A tuneable laser (HP-Agilent, 8168-F) with a wavelength range of 1450-1590 nm, a typical output power of $6 \pm 1.4 \mathrm{~mW}$, FWHM of $4 \mathrm{~nm}$ (spectral resolutionlimited), and a collimated beam width of $4.2 \mathrm{~mm}$ was employed for EQE measurements. The excitation power density was characterized using a calibrated germanium photodiode (Newport, 818-IR) and a NIR camera (Electrophysics, Micronviewer 7290A), while the photogenerated current was measured with a source-meter (Keithley Instruments, 2440-C). More experimental details on the experimental setup can be found in a previous work [23]. The solar concentration $C_{e x c}$ was calculated as [23]:

$$
C_{\text {exc }}=\frac{\int P_{\text {exc }}(\lambda) \partial \lambda}{\int P_{\mathrm{AM} 1.5 D}(\lambda) \partial \lambda}
$$


, where $P_{e x c}$ is the excitation power density or irradiance at the respective wavelength, $P_{A M 1.5 D}$ the spectral irradiance of the air-mass 1.5 direct (AM1.5D) solar spectrum [37] between $\lambda_{1}$ and $\lambda_{2}$, the integration limits corresponding to the absorption band of the up-converter.

The up-converter solar cells were based on planar bifacial solar cells fabricated on c-Si wafers, with a double-layer anti-reflection coating (ARC) - comprised of $110 \mathrm{~nm}$ hydrogenated silicon nitride (a$\left.\mathrm{SiN}_{\mathrm{x}}: \mathrm{H}\right)$ and $110 \mathrm{~nm}$ magnesium fluoride $\left(\mathrm{MgF}_{2}\right)$ - on the front and a single-layer ARC of $120 \mathrm{~nm}$ a$\mathrm{SiN}_{\mathrm{x}}: \mathrm{H}$ on the rear. The infrared absorptivity of silicon solar cells is highly sensitive to the texture angle. Riverola et.al. reported the dramatic reduction of $\lambda=1-6 \mu \mathrm{m}$ absorptivity of silicon when the texture angle is reduced from $60^{\circ}$ to $0^{\circ}$ [38]. These results were confirmed in the results of Rudiger et.al [36] comparing the transmittance and reflectance of planar and textured solar cells. In a planar solar cell, the infrared transmittance increased more than 20\% compared to the textured cell, as shown in Figure 2. As a result, trapping of infrared photons in the cell was reduced. Without this trapping, however, reflection increased in the visible, reducing the EQE of the planar cell between 300-1200 nm as shown below.

Therefore, there is a trade-off between increasing infrared photons for the upconverter, while reducing visible photons and finally the photo-current of the solar cell. In a practical solar cell with texture, a lower infrared transmission will result to reduced upconverted light. At 1 sun, the $\Delta \mathrm{J}_{\mathrm{sc}}$ from the sacrificial move from texturized to planar solar cell is $42.86-34.15=8.71 \mathrm{~mA} / \mathrm{cm}^{2}$. However, the record $\Delta \mathrm{Jsc}$ due to state-of-the-art upconverters was characterized at 94 suns. At this solar concentration the current density by the integral of the AM1.5D spectrum results to $357 \mathrm{~mA} / \mathrm{cm}^{2}$. A device based on the planar solar cells with solar concentration atop of the solar cell should produce at least a $\Delta \mathrm{Jsc}$ of 357 $42=315 \mathrm{~mA} / \mathrm{cm}^{2}$ in the infrared to break even the sacrifices in the visible. At 94 suns the benefit from the upconverter is more than one order of magnitude lower than the sacrifice of the texture-to-planar solar cell. Consequently, combinations of different technologies in addition to solar concentration are required to enhance the performance of upconverters [14].

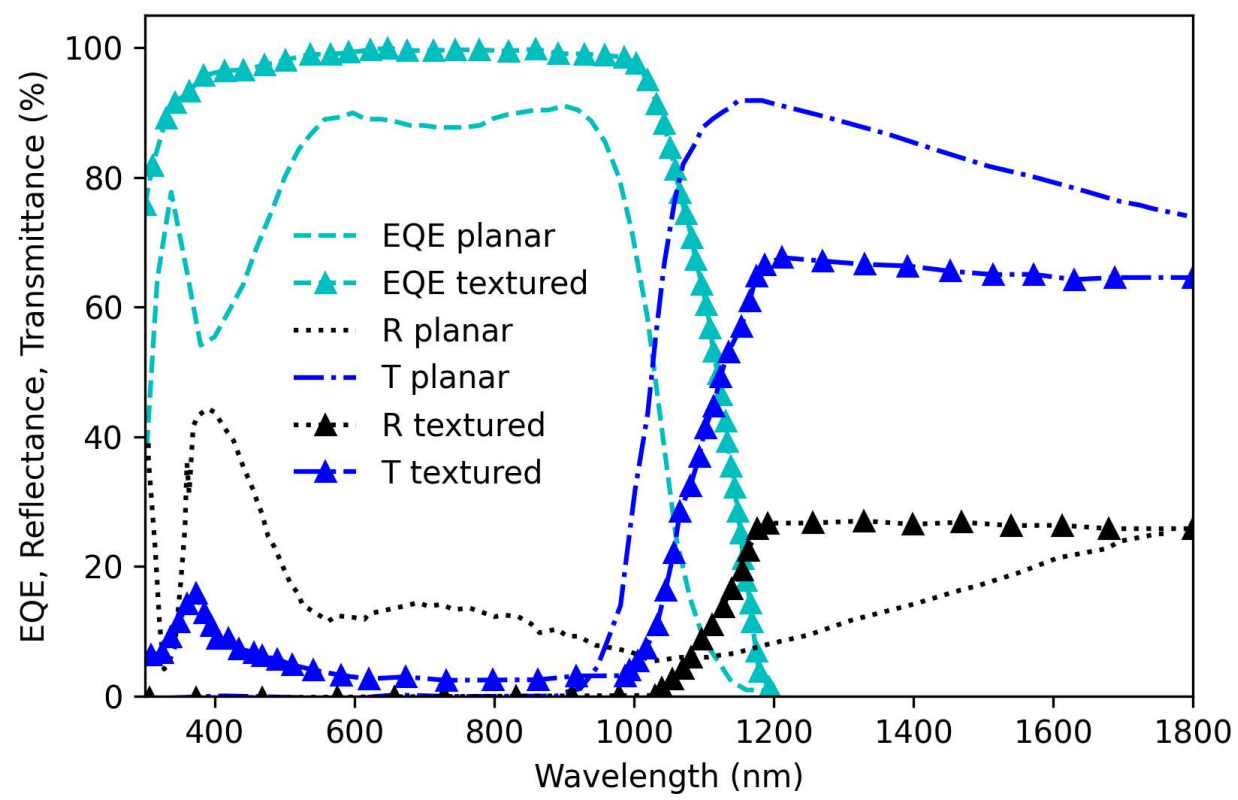

Figure 2: EQE, reflectance and transmittance of the planar bifacial solar cells utilized in the devices. Reflectance and transmittance was measured before metallization. Adapted from [36,39] 
The absorptivity of silicon at $\lambda=1-6 \mu \mathrm{m}$ is proportional to the concentration of the emitter and the BSF from $5 \times 10^{17}$ to $5 \times 10^{20} \mathrm{~cm}^{-3}$ [38]. At a sheet resistance (Rsh) of $62 \Omega / \mathrm{sq}$, a maximum phosphorus concentration of $3.3 \times 10^{20} \mathrm{~cm}^{-3}$ can increase the absorption of a textured silicon wafer by approximately $40 \%$ [40]. An intuitive development towards reduced absorptivity of free carriers was reported in [36] via localized BSF centered near the highly reflecting contacts. Despite the low $\mathrm{Rsh}=19 \Omega / \mathrm{sq}$ of the BSF, the transmittance of the cells, did not show significant improvements. A boron-doped emitter profile with high sheet resistance of Rsh-130 $\Omega /$ sq was utilized, which may be associated with a high concentration of free carriers at the front side of the cells further limiting the infrared transmission. For further details on the optimization of the solar cells the reader is directed towards the work by [36].

$\mathrm{BaY}_{2} \mathrm{~F}_{8}: 30 \% \mathrm{Er}^{3+}$ crystals were prepared under high purity argon conditions via the Czochralski process. Fluoride powders of $99.999 \%$ purity were melted at $972^{\circ} \mathrm{C}$ in a custom-built furnace, evacuated to $10^{-}$ ${ }^{7}$ mbar to minimize contamination [31]. Finally, the samples were cut in rectangular blocks and polished to optical quality. The dimensions of the polished up-converter utilized here was $10.08 \mathrm{~mm} \times 10.03 \mathrm{~mm} \times 2.33 \mathrm{~mm}$. The absorption coefficients were measured in an ultraviolet-visibleNIR spectrophotometer (Perkin-Elmer, Lambda 950).

\section{Results and Discussion}

\subsection{Localized irradiance in the up-converter}

The integrated optics concentrate light and locally change the distribution of the irradiance at the surface of the up-converter to improve the performance of the solar cell device [23]. In addition to the irradiance distribution at the surface of the up-converter, it is important to know the distribution of light inside a mono-crystalline up-converter and how this may affect its performance. The simulated local irradiance at $1493 \mathrm{~nm}$ at $210 \mathrm{~W} / \mathrm{m}^{2}$ as a function of depth $Z$ in the up-converter can be seen in Figure 3 for the three cases illustrated in Figure 1(a-c). The absorption coefficient of the up-converter (plotted in Figure 5) was considered in this simulation. The irradiance of the reference exhibits a log-normal distribution with the peak at $30 \mu \mathrm{m}$. The irradiance of $\mathrm{CPC}-25^{\circ}$ and $\mathrm{CPC}-45^{\circ}$, in addition to the same log-normal distribution, exhibit additional peaks at $Z=370 \mu \mathrm{m}$ and $Z=720 \mu \mathrm{m}$, proportional to the focal lengths of the CPC. A fraction of the light is transmitted without concentration by the CPC, resulting in a log-normal distribution. Excitation light reflected at the side walls of the parabola, see Figure 1(c-d), will be focused inside the up-converter depending on its focal length. The focal length of the $\mathrm{CPC}-45^{\circ}$ resides $720 \mu \mathrm{m}$ inside the up-converter, while the absorption coefficient of $\mathrm{BaY}_{2} \mathrm{~F}_{8}$ : $30 \% \mathrm{Er}^{3+}$ at $1493 \mathrm{~nm}$ is $44 \mathrm{~cm}^{-1}$. Therefore an amount of $1493 \mathrm{~nm}$ excitation will be absorbed before the focus. This dilute, compared to the laser beam, excitation, will be up-converted to visible wavelengths, as shown in the photographs of Figure 3. Due to this dilution and hence lower excitation irradiance to the reference beam, process ETU2 with emission at $650 \mathrm{~nm}$ may be more probable than ETU3 with emission at $550 \mathrm{~nm}$, as qualitatively evidenced in the photograph. The irradiance profile of the concave reflector (not shown) is also estimated to have log-normal distribution with intensity proportional to $\mathrm{e}^{-}$ $\mathrm{a} 2 \mathrm{z} / \cos \theta$ 

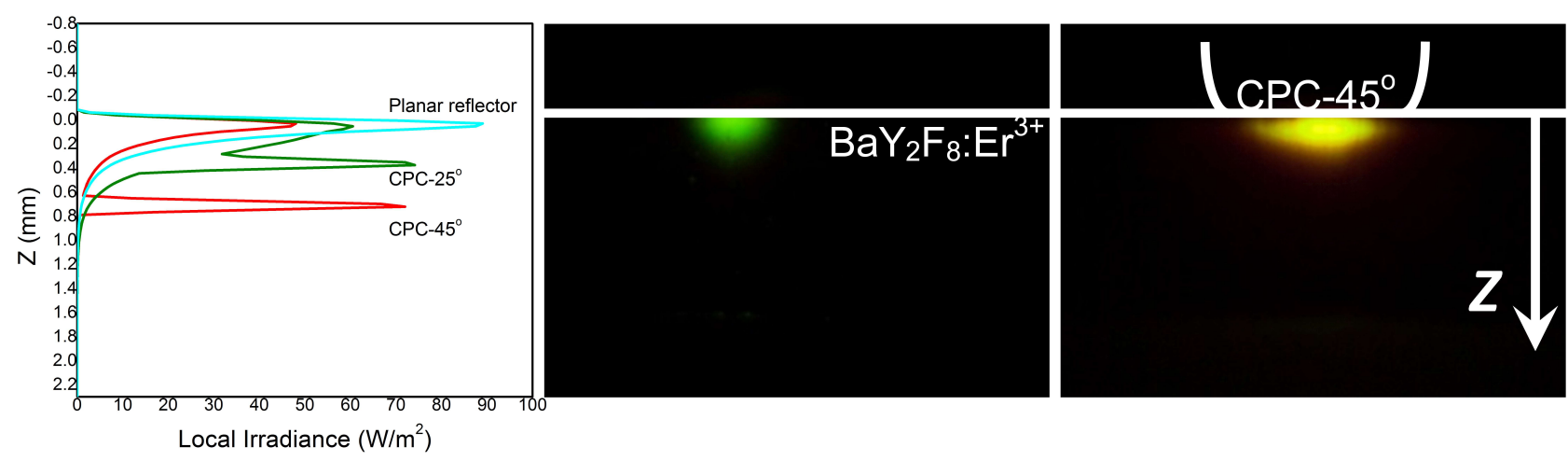

Figure 3: Simulated irradiance profile in the up-converter along the $\mathrm{Z}$ axis at the centre of the excitation beam. The CPC: up-converter interface is at $0 \mathrm{~mm}$. Photographs of the reference (left) and CPC-45 (right) illuminated by $1493 \mathrm{~nm}$ light are also displayed inline.

The secondary peaks of CPC- $25^{\circ}$ and $\mathrm{CPC}-45^{\circ}$ exhibit similar maximum irradiance, lower than that of the reference. The localized excitation peaks, correspond to centers with higher irradiance and consequently iPLQY. This result agrees with a higher iPLQY and ePLQY being measured for a thinner $\mathrm{BaY}_{2} \mathrm{~F}_{8}: 30 \% \mathrm{Er}^{3+}$ sample [29], whereby a $1.76 \mathrm{~mm}$-thick sample exhibited a higher probability of upconverted light being externally emitted. This is largely because a thinner sample suffers reduced reabsorption losses. For the investigated $\mathrm{BaY}_{2} \mathrm{~F}_{8}: 30 \% \mathrm{Er}^{3+}$ up-converter, the re-absorption losses have been estimated to be as high as $51 \%$ [29].

The irradiance of emission at $970 \mathrm{~nm}$ at top of the up-converter interface that is directed to the solar cell (see Figure 1a-c) was simulated and shown in Figure 4. The emission is assumed to be a result of the excitation at $1493 \mathrm{~nm}$ at top of the up-converter. Therefore, it was simulated with the same illumination conditions, direct or concentrated (reference or CPC) illumination.

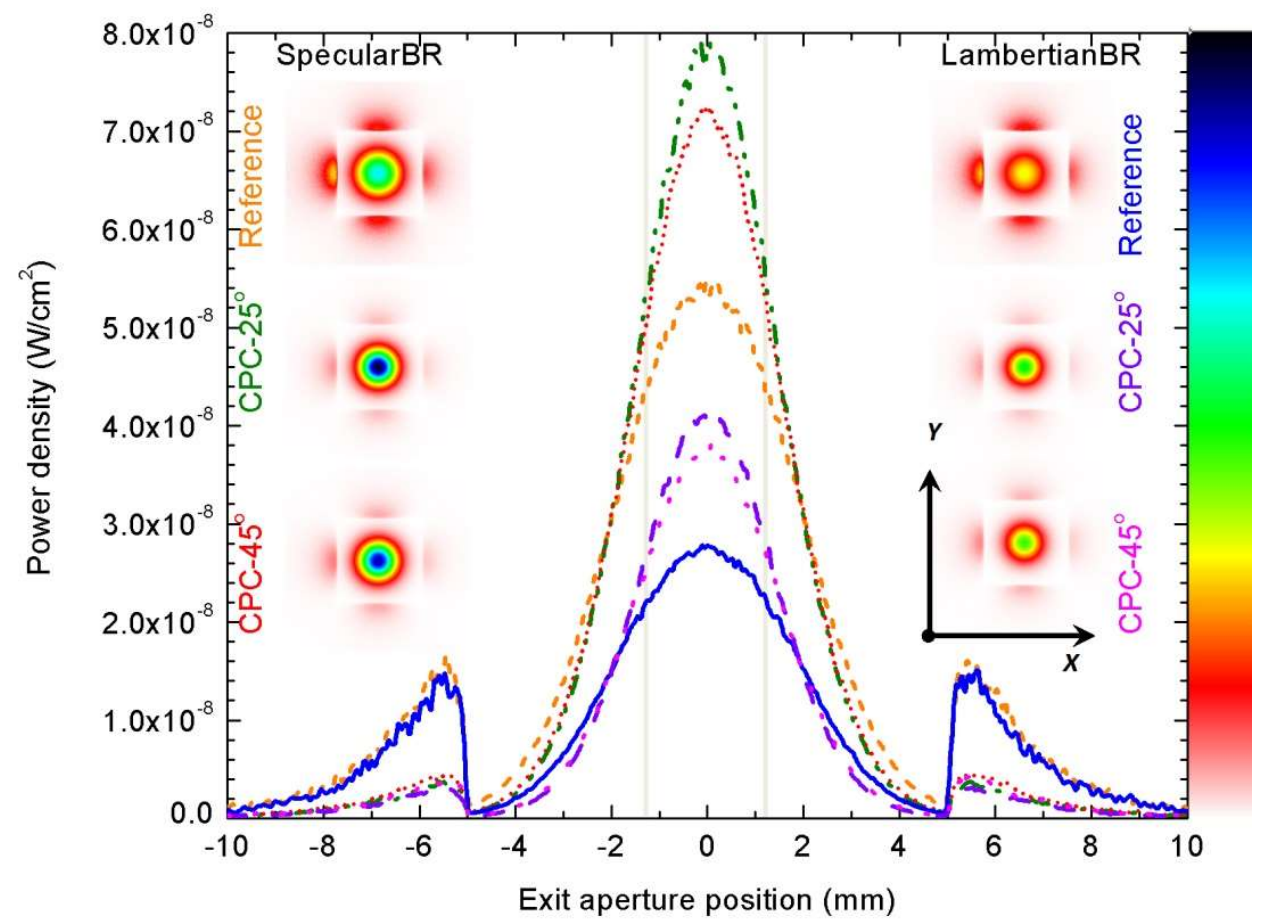

Figure 4: Irradiance profiles of the emission at $970 \mathrm{~nm}$ at the $\mathrm{CPC}$ : up-converter interface after reflection, simulated by ray-tracing. Six configurations are displayed for the reference (... and - ), CPC- $25^{\circ}$ (..-.. and - - ) and CPC- $45^{\circ}(--$ and - -) with specular and Lambertian reflectors, respectively. The insets expose the spatial distribution of the irradiance transmitted out of the up-converter, with corresponding colours on the right. 
The emission exhibits an irradiance profile consisting of one central peak as a result of the excitation beam and two lateral peaks outside the $10 \times 10 \mathrm{~mm}$ up-converter ( $\pm 5 \mathrm{~mm}$ of aperture position in $\mathrm{X}$ axis). The lateral peaks are higher for the reference than both CPC as a result of a wider illuminated area, $4.2 \mathrm{~mm}$ compared to $2.5 \mathrm{~mm}$. Consequently, more $970 \mathrm{~nm}$ emission laterally escapes the up-converter in the reference compared to the CPCs. The intensity is higher for the specular back reflector than the Lambertian in all cases. This is a result of the Lambertian inducing a longer path-length in the upconverter and therefore higher absorption in the up-converter.

The emission can also be affected by the back reflector whether specular or Lambertian, however, for highly absorbing up-converters the contribution of this effect to $\mathrm{EQE}$ is expected to be negligible. Consequently, light emitted near the top of the up-converter and collected by the overlying solar cell should lead to a higher EQE which is presented in the next section.

\subsection{External quantum efficiency - wavelength dependence}

Figure 5 displays the EQE of the investigated configurations illuminated at a constant power density of $90 \mathrm{~W} / \mathrm{m}^{2}$ or $2.95 \times$ concentrated atop the devices, the maximum available with our laser across the measured spectrum.

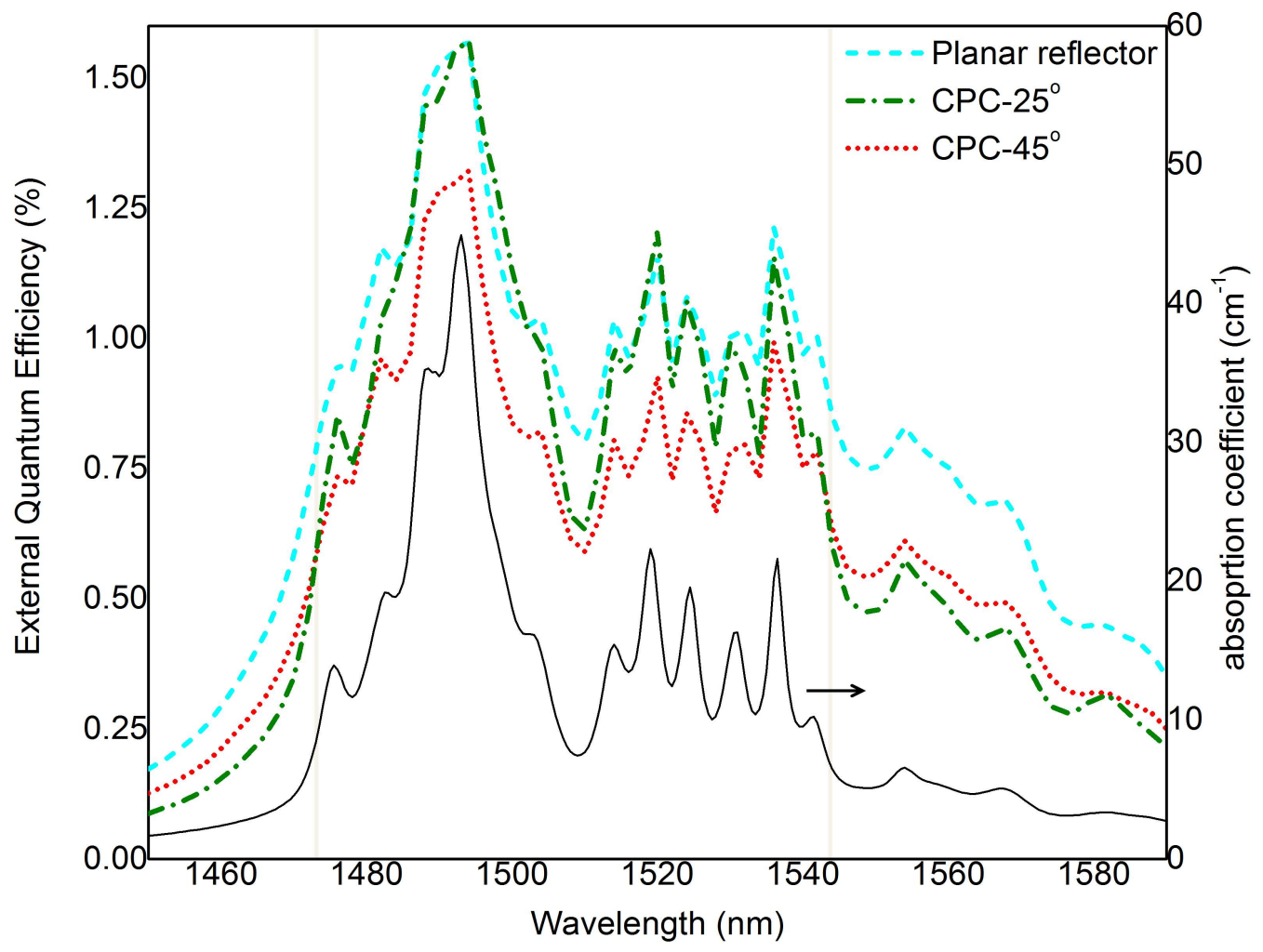

Figure 5: Measured EQE spectra of the UC-SC measured at $90 \mathrm{~W} / \mathrm{m}^{2}$ with: a) reference, b) $\left.\mathrm{CPC}-45^{\circ} \mathrm{c}\right) \mathrm{CPC}-25^{\circ}$ geometries. The absorption tails of the up-converter are indicated by gray vertical lines.

The shape of all three EQE spectra closely match the absorption coefficient of $\mathrm{BaY}_{2} \mathrm{~F}_{8}: 30 \% \mathrm{Er}^{3+}$, reproducing the Stark structure of the ${ }^{4} I_{15 / 2}-{ }^{4} I_{13 / 2}$ levels. This structure exhibits evidence of energy transfer up-conversion (ETU) as the dominant UC mechanism [41] common in highly $\mathrm{Er}^{3+}$-doped materials [42]. The concentration of $\mathrm{Er}^{3+}$ is $30 \%$ which promotes ETU and precludes excited state absorption from being the dominant population mechanism. The EQE of case a) reference and c) CPC$25^{\circ}$ is higher than b) CPC- $45^{\circ}$ between $1472-1542 \mathrm{~nm}$. Only in the absorption tails region $(\lambda<1472 \mathrm{~nm}$ 
and $\lambda>1542 \mathrm{~nm}$, indicated by gray vertical lines) is the EQE using the $\mathrm{CPC}-45^{\circ}$ fractionally higher than CPC-25.

The EQE is the ratio of photo-generated carriers to the incident NIR photons. The obtained spectra can be explained in relation to the wavelength-dependent infrared absorptance and the path-length of the up-converter, displayed in Figure 6. At $\lambda<1472 \mathrm{~nm}$ and $\lambda>1542 \mathrm{~nm}$, the low absorptance leads to different path-lengths in each case. CPC- $25^{\circ}$ induces the longest path-length, which is higher than CPC$45^{\circ}$ and the reference. $\mathrm{CPC}-45^{\circ}$ converges excitation light at an angle $\theta=35.3^{\circ}$ (see Figure 1d). At this angle, excitation light travels on average $1 \mathrm{~cm}$ longer path-length than the reference. However, in CPC$25^{\circ}$ with an angle $\theta=66.85^{\circ}$ (see Figure 1b) excitation light has two-times longer path-length in the up-converter.

At the band $1472-1542 \mathrm{~nm}$, however, the absorptance is $>90 \%$ and the path-length is equal in all cases. It can be seen that the EQE of each configuration is proportional to the depth $\mathrm{Z}$ from the CPC: crystal interface. In the devices with planar back reflectors, the closer these high intensity regions are to the solar cell, the higher the collection and therefore the EQE will be. Upconversion is emitted spontaneously in a $4 \pi$ solid angle. Depending on the path-length, several re-absorption events will reduce the upconversion externally emitted from the sample. Consequently, the high intensity excitations occurring near the surface and close to the solar cell, will propagate a shorter pathlength before being absorbed by the solar cell, resulting to a higher EQE. Consequently, the performance of mono-crystalline up-converter solar cells can be higher when the excitation is accumulated near the top surface of the up-converter. The absorptance of the concave mirror is also $>90 \%$ at $1472-1542 \mathrm{~nm}$, while lower than the CPC-25 and higher than the CPC-45 and reference outside this band. Contrary to the CPC, the focus of the concave mirror can be adjusted for higher performance near the top surface of the up-converter and is presented in the next section.
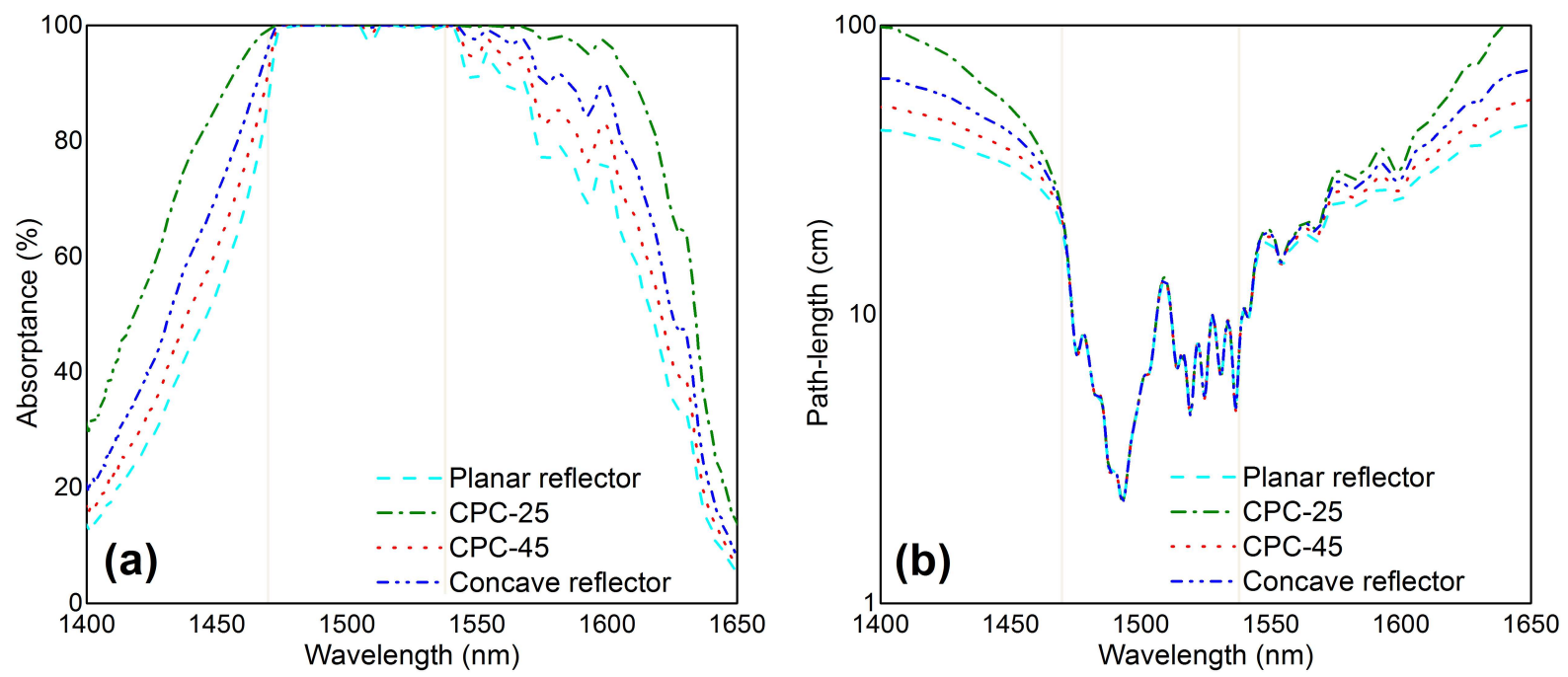

Figure 6: Absorptance (a) and path-length (b) of reference and CPC cases as a function of wavelength. The absorption tails of the up-converter are indicated by gray vertical lines.

\subsection{Quantum efficiency - power dependence}

The quantum efficiency was further characterized at the peak wavelength of $1493 \mathrm{~nm}$ as a function of power density and solar concentration. Figure 7(a) displays the EQE as a function of the power density incident on the device, while in Figure 7(b) the internal quantum efficiency (IQE) is displayed incorporating the transmittance through the c-Si solar cell and the concentrators.

At a low power density, the EQE of $\mathrm{CPC}-25^{\circ}$ is higher than $\mathrm{CPC}-45^{\circ}$, planar and concave reflector devices. At power density higher than $90 \mathrm{~W} / \mathrm{m}^{2}$, the planar device performs better than $\mathrm{CPC}-25^{\circ}$ and 
$\mathrm{CPC}-45^{\circ}$. This is likely due to peripheral emission losses in the CPC devices, see Figure 4. As shown in Figure 6(a), absorptance at $1493 \mathrm{~nm}$ is higher than $99 \%$ in all devices and aligns with a large fraction of absorbed pump power [43]. In the planar device, the absorbed power is concentrated at a shorter path-length in the up-converter as shown in Figure 3. The performance of the concave reflector device is lower than the CPC devices below $340 \mathrm{~W} / \mathrm{m}^{2}$ but higher above this power density. Excitation in the concave reflector device undergoes twice the path-length of the CPC devices. A longer pathlength increases the re-absorption in the upconverter. As a result, the excitation intensity reaching the reflector is reduced, similar to emission at visible wavelengths in the photographs of Figure 3. Consequently, below $340 \mathrm{~W} / \mathrm{m} 2$ the measured EQE results from upconverted light near the surface, while above this power density a higher external upconversion quantum yield (ePLQY) [12] results to more upconversion reaching the solar cell. Up-converted light as well as excitation is concentrated back to the up-converter by the concave reflector and finally absorbed by the solar cell. After taking into account the transmittance of the solar cell and the concentrators, the IQE of the devices is obtained with the highest IQE of $7.2 \%$ at $876 \mathrm{~W} / \mathrm{m}^{2}$ in the device with a concave reflector. The power conversion efficiency of the bifacial silicon cells increased by $0.19 \%$ under infrared excitation. The silicon cells without upconverter had an efficiency of 16.7\% under AM1.5G conditions [27].

Several device effects are incorporated in the quantum efficiency in addition to a photoluminescence intensity measurement. These effects are: i) the spectral response of the solar cell absorbing upconversion from all energy levels despite that higher than $90 \%$ is from the ${ }^{4} I_{11 / 2}$ level, ii) the transmission of the solar cell at 1400-1600 nm, iii) a double path-length through the up-converter, and iv) the angular extent of the concentrators leading to variable local irradiance in the up-converter as presented in section 3.1 and further peripheral losses. The losses around the edges of the up-converter can be minimized by peripheral reflectors, as implemented with aluminized mylar [31]. It was demonstrated here that better performance obtains with concave reflectors in place of planar back reflectors. The used Ag-coated concave reflector has reduced reflectance around $1000 \mathrm{~nm}$, thereby a higher IQE can be expected by using Au-coated reflectors.
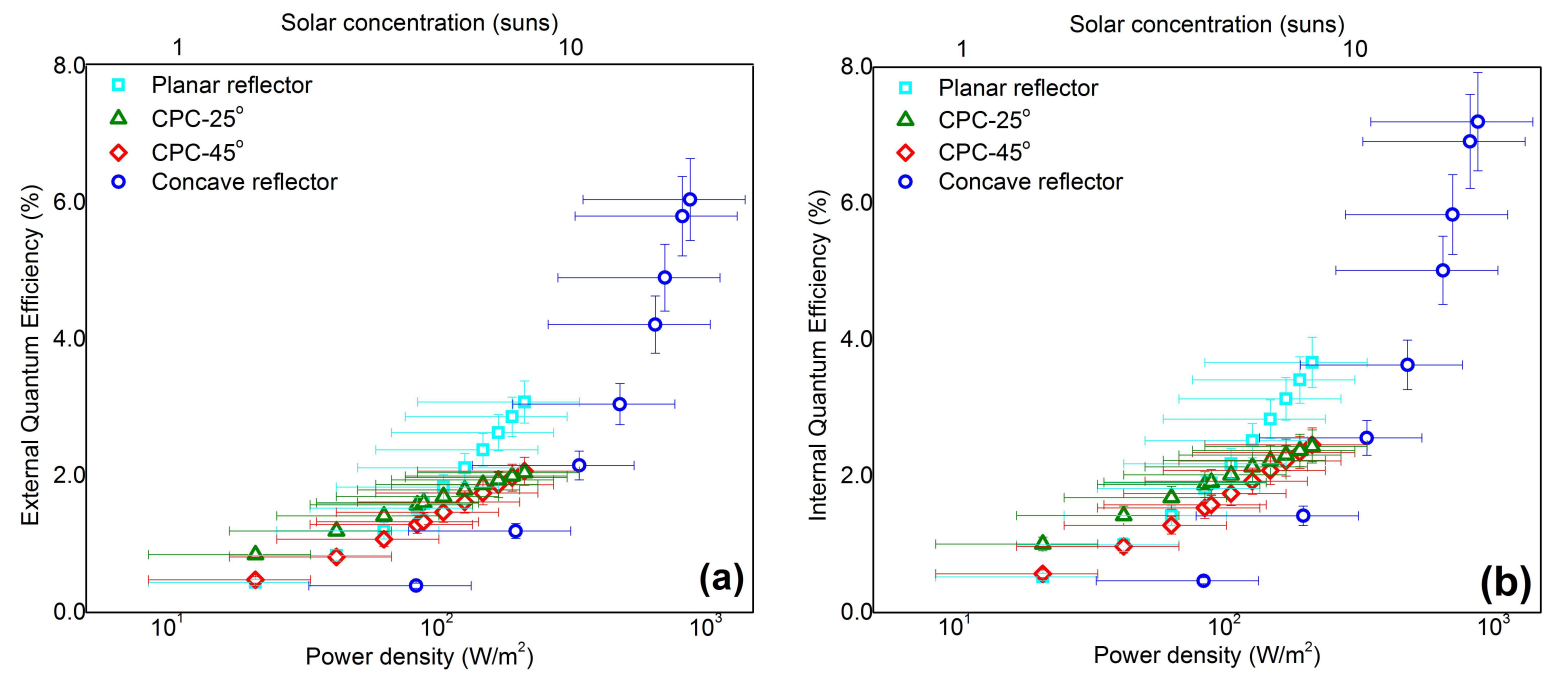

Figure 7: (a) EQE and (b) IQE of the up-conversion solar cell devices excited at $1493 \mathrm{~nm}$ as a function of incident power density atop the devices and equivalent solar concentration on semi-logarithmic scales.

The results suggest there is space for better performance from devices implementing higher concentration reflectors with mono-crystalline up-converters. Practical up-scaling of the integrated optics is promising given the practical advances in bifacial solar panels. To permit wider application, 1sun operation atop of the devices is accommodating. Consequently, a concentration factor at least in the 
order of 30x, as evidenced in this report, can be accommodated by advances in micro-optics with ability for wide area application. CPC of acceptance angle less than $25^{\circ}$ and $100 \mu \mathrm{m}$ exit aperture [23] with shorter focal length will result in localized irradiance accumulated near the up-converter and a higher collection probability by the solar cell. A low concentration of $2.8 \times$ was attained with the presented concentrators. Higher solar concentration under sunlight is achieved in parabolic aplanat systems with secondary concentration. In these experiments, upconverters were characterized under solar concentration of 2300 suns [44]. In addition to increased quantum yield at higher concentration, a broader excitation can populate additional transitions in upconverters with potential for higher quantum yields [45].

Imaging optics would be able to concentrate to a focal spot near the diffraction limit, however, require high throughput and improved collection efficiency [22,46]. Optics developed for biomedical applications and bio-sensing with simultaneous capabilities of concentration and efficient collection can also be suitable to this end [24]. Specifically, dielectric microbeads [25] and microlens arrays $[47,48]$ have demonstrated several orders of magnitude enhanced photonic devices, whereas double layer metasurfaces [49] exhibited enhancements by tailored confinement at the excitation and emission wavelengths.

\section{Conclusions}

The performance of mono-crystalline up-converter solar cell device tandems with integrated optics was presented in this paper. Solar cell devices coupled to a CPC with a lower acceptance angle $\left(\mathrm{CPC}-25^{\circ}\right)$ exhibit higher IQE than the planar and CPC $-45^{\circ}$ below $1493 \mathrm{~nm}$ excitation at $90 \mathrm{~W} / \mathrm{m}^{2}$. At higher power density the CPC devices exhibit lower performance affected by the focal length of the CPC, which is shorter for the $\mathrm{CPC}-25^{\circ}$ compared to the $\mathrm{CPC}-45^{\circ}$. In the $\mathrm{CPC}$ the local irradiance is accumulated $370 \mu \mathrm{m}-720 \mu \mathrm{m}$ deeper in the up-converter, while in the planar device the irradiance is distributed near the top surface of the up-converter which is optically coupled to the solar cell, peaking at $30 \mu \mathrm{m}$. The highest IQE of $7.2 \%$ was measured with a concave reflector at $876 \mathrm{~W} / \mathrm{m}^{2}$ concentrating transmitted excitation and emission from the up-converter back to the solar cell. By knowing the irradiance distribution in the mono-crystalline up-converter and how this affects the performance of upconversion solar cells, further improvements can be achieved by microspheric tailored optics as recently reported $[25,47]$ and optimized up-converters with reduced re-absorption.

\section{Acknowledgements}

This research received the financial support of the Engineering and Physical Sciences Research Council (EPSRC) No. EP/I013245/1, the European Community's Seventh Framework Program (FP7/2007-2013) No. [246200] and the European Cooperation of Scientific \& Technical Research (COST) via action CM1403. The authors wish to thank Jan C. Goldschmidt and Stefan Fischer from Fraunhofer Institute for Solar Energy Systems (Freiburg, Germany) for providing the silicon solar cell used in this work and the anonymous reviewers for providing valuable and constructive comments considerably improving this work. B.S.R. would like to acknowledge research funding from the Helmholtz Association: i) a Recruitment Initiative fellowship; and ii) the Research Field Energy Program Materials and Technologies for the Energy Transition - Topic 1 Photovoltaics.

\section{References}

[1] B.S. Richards, Enhancing the performance of silicon solar cells via the application of passive luminescence conversion layers, Solar Energy Materials and Solar Cells. 90 (2006) 2329-2337. https://doi.org/10.1016/j.solmat.2006.03.035.

[2] Ashwin C. Atre, J.A. Dionne, Realistic upconverter-enhanced solar cells with non-ideal absorption and recombination efficiencies, Journal of Applied Physics. 110 (2011) 034505. https:// doi.org/10.1063/1.3610522. 
[3] C.M. Johnson, G.J. Conibeer, Limiting efficiency of generalized realistic c-Si solar cells coupled to ideal up-converters, Journal of Applied Physics. 112 (2012) 103108.

https://doi.org/10.1063/1.4766386.

[4] Photovoltaics Report - Fraunhofer ISE, Fraunhofer Institute for Solar Energy Systems ISE. (n.d.). http://www.ise.fraunhofer.de/en/publications/studies/photovoltaics-report.html.

[5] T.M. Bruton, K.C. Heasman, J.P. Nagle, R.R. Russell, The achievement of $20 \%$ efficiency in a CZ silicon solar cell under concentration, in: Conference Record of the Twenty Third IEEE Photovoltaic Specialists Conference - 1993 (Cat. No.93CH3283-9), 1993: pp. 1250-1251. https:// doi.org/10.1109/PVSC.1993.346942.

[6] M. Vivar, C. Morilla, I. Antón, J.M. Fernández, G. Sala, Laser grooved buried contact cells optimised for linear concentration systems, Solar Energy Materials and Solar Cells. 94 (2010) 187-193. https://doi.org/10.1016/j.solmat.2009.08.022.

[7] A. Slade, V. Garboushian, 27.6\% efficient silicon concentrator solar cells for mass production, in: Technical Digest, 15th International Photovoltaic Science and Engineering Conference, Beijing, 2005.

[8] M.A. Green, Y. Hishikawa, E.D. Dunlop, D.H. Levi, J. Hohl-Ebinger, A.W.Y. Ho-Baillie, Solar cell efficiency tables (version 52), Progress in Photovoltaics: Research and Applications. 26 (2018) 427-436. https://doi.org/10.1002/pip.3040.

[9] A.J. McEvoy, L. Castañer, T. Markvart, Solar Cells: Materials, Manufacture and Operation, Academic Press, 2012.

[10] P. Campbell, M.A. Green, The limiting efficiency of silicon solar cells under concentrated sunlight, IEEE Transactions on Electron Devices. 33 (1986) 234-239.

[11] A. Ivaturi, S.K.W. MacDougall, R. Martín-Rodríguez, M. Quintanilla, J. Marques-Hueso, K.W. Krämer, A. Meijerink, B.S. Richards, Optimizing infrared to near infrared upconversion quantum yield of $\beta$-NaYF4:Er3+ in fluoropolymer matrix for photovoltaic devices, Journal of Applied Physics. 114 (2013) 013505. https://doi.org/10.1063/1.4812578.

[12] A. Boccolini, J. Marques-Hueso, B.S. Richards, Self-absorption in upconverter luminescent layers: impact on quantum yield measurements and on designing optimized photovoltaic devices, Opt. Lett., OL. 39 (2014) 2904-2907. https://doi.org/10.1364/OL.39.002904.

[13] R. Martín-Rodríguez, S. Fischer, A. Ivaturi, B. Froehlich, K.W. Krämer, J.C. Goldschmidt, B.S. Richards, A. Meijerink, Highly efficient IR to NIR upconversion in Gd2O2S: Er3+ for photovoltaic applications, Chem. Mater. 25 (2013) 1912-1921. https://doi.org/10.1021/cm4005745.

[14] B.S. Richards, D. Hudry, D. Busko, A. Turshatov, I.A. Howard, Photon Upconversion for Photovoltaics and Photocatalysis: A Critical Review, Chem. Rev. 121 (2021) 9165-9195. https://doi.org/10.1021/acs.chemrev.1c00034.

[15] S.K.W. MacDougall, A. Ivaturi, J. Marques-Hueso, K.W. Krämer, B.S. Richards, Broadband photoluminescent quantum yield optimisation of Er3+-doped $\beta$-NaYF4 for upconversion in silicon solar cells, Solar Energy Materials and Solar Cells. 128 (2014) 18-26. https://doi.org/10.1016/j.solmat.2014.05.004.

[16] R.E. Joseph, C. Jiménez, D. Hudry, G. Gao, D. Busko, D. Biner, A. Turshatov, K. Krämer, B.S. Richards, I.A. Howard, Critical Power Density: A Metric To Compare the Excitation Power Density Dependence of Photon Upconversion in Different Inorganic Host Materials, J. Phys. Chem. A. 123 (2019) 6799-6811. https://doi.org/10.1021/acs.jpca.9b03851.

[17] R.E. Joseph, D. Busko, D. Hudry, G. Gao, D. Biner, K. Krämer, A. Turshatov, B.S. Richards, I.A. Howard, A method for correcting the excitation power density dependence of upconversion emission due to laser-induced heating, Optical Materials. 82 (2018) 65-70. https://doi.org/10.1016/j.optmat.2018.05.025. 
[18] Y. Takeda, S. Mizuno, H.N. Luitel, T. Tani, A broadband-sensitive upconverter: Garnet-type Ca3Ga2Ge3O12 codoped with Er3+, Y3+, Li+, Ni2+, and Nb5+, Journal of the American Ceramic Society. 102 (2019) 3457-3467. https://doi.org/10.1111/jace.16193.

[19] J. Marques-Hueso, R. Peretti, R. Abargues, B.S. Richards, C. Seassal, J.P. Martínez-Pastor, Photonic crystal-driven spectral concentration for upconversion photovoltaics, Advanced Optical Materials. 3 (2015) 568-574. https://doi.org/10.1002/adom.201400402.

[20] J.C. Goldschmidt, S. Fischer, Upconversion for photovoltaics - a review of materials, devices and concepts for performance enhancement, Advanced Optical Materials. 3 (2015) 510-535. https://doi.org/10.1002/adom.201500024.

[21] J. Pedrini, A. Monguzzi, Recent advances in the application triplet-triplet annihilation-based photon upconversion systems to solar technologies, JPE, JPEOBV. 8 (2017) 022005. https://doi.org/10.1117/1.JPE.8.022005.

[22] G.E. Arnaoutakis, J. Marques-Hueso, A. Ivaturi, K.W. Krämer, S. Fischer, J.C. Goldschmidt, B.S. Richards, Enhanced up-conversion for photovoltaics via concentrating integrated optics, Opt. Express, OE. 22 (2014) A452-A464. https://doi.org/10.1364/OE.22.00A452.

[23] G.E. Arnaoutakis, J. Marques-Hueso, A. Ivaturi, S. Fischer, J.C. Goldschmidt, K.W. Krämer, B.S. Richards, Enhanced energy conversion of up-conversion solar cells by the integration of compound parabolic concentrating optics, Solar Energy Materials and Solar Cells. 140 (2015) 217-223. https://doi.org/10.1016/j.solmat.2015.04.020.

[24] G.E. Arnaoutakis, B.S. Richards, Geometrical concentration for enhanced up-conversion: A review of recent results in energy and biomedical applications, Optical Materials. 83 (2018) 4754. https://doi.org/10.1016/j.optmat.2018.05.064.

[25] L. Liang, D.B.L. Teh, N.-D. Dinh, W. Chen, Q. Chen, Y. Wu, S. Chowdhury, A. Yamanaka, T.C. Sum, C.-H. Chen, N.V. Thakor, A.H. All, X. Liu, Upconversion amplification through dielectric superlensing modulation, Nat Commun. 10 (2019) 1391. https://doi.org/10.1038/s41467-01909345-0.

[26] S. Fischer, R. Martín-Rodríguez, B. Fröhlich, K.W. Krämer, A. Meijerink, J.C. Goldschmidt, Upconversion quantum yield of Er3+-doped $\beta$-NaYF4 and Gd2O2S: The effects of host lattice, Er3+ doping, and excitation spectrum bandwidth, Journal of Luminescence. 153 (2014) 281-287. https://doi.org/10.1016/j.jlumin.2014.03.047.

[27] S. Fischer, A. Ivaturi, B. Fröhlich, M. Rüdiger, A. Richter, K.W. Krämer, B.S. Richards, J.C. Goldschmidt, Upconverter silicon solar cell devices for efficient utilization of sub-band-gap photons under concentrated solar radiation, IEEE Journal of Photovoltaics. 4 (2014) 183-189. https://doi.org/10.1109/JPHOTOV.2013.2282744.

[28] S. Fischer, A. Ivaturi, P. Jakob, K.W. Krämer, R. Martin-Rodriguez, A. Meijerink, B. Richards, J.C. Goldschmidt, Upconversion solar cell measurements under real sunlight, Optical Materials. 84 (2018) 389-395. https://doi.org/10.1016/j.optmat.2018.05.072.

[29] A. Boccolini, E. Favilla, M. Tonelli, B.S. Richards, R.R. Thomson, Highly efficient upconversion in Er3+doped BaY2F8 single crystals: dependence of quantum yield on excitation wavelength and thickness, Opt. Express, OE. 23 (2015) A903-A915. https://doi.org/10.1364/OE.23.00A903.

[30] S. Fischer, E. Favilla, M. Tonelli, J.C. Goldschmidt, Record efficient upconverter solar cell devices with optimized bifacial silicon solar cells and monocrystalline BaY2F8:30\% Er3+ upconverter, Solar Energy Materials and Solar Cells. 136 (2015) 127-134. https://doi.org/10.1016/ j.solmat.2014.12.023.

[31] A. Boccolini, R. Faoro, E. Favilla, S. Veronesi, M. Tonelli, BaY2F8 doped with Er3+: An upconverter material for photovoltaic application, Journal of Applied Physics. 114 (2013) 064904. https://doi.org/10.1063/1.4817171.

[32] A.A. Kaminskii, O. Lux, J. Hanuza, H. Rhee, H.J. Eichler, J. Zhang, D. Tang, D. Shen, H. Yu, J. Wang, H. Yoneda, A Shirakawa, Monoclinic $\beta$-BaY 2 F 8 -a novel crystal simultaneously active 
for SRS and Ln 3+ -ion lasing, Laser Phys. 25 (2015) 015801. https://doi.org/10.1088/1054-660X/ 25/1/015801.

[33] J. Mistrik, I. Ohlidal, R. Antos, M. Aoyama, T. Yamaguchi, Evidence of refractive index change in glass substrates induced by high-density reactive ion plating deposition of SiO2 films, Applied Surface Science. 244 (2005) 51-54. https://doi.org/10.1016/j.apsusc.2004.09.128.

[34] J. Gibbons, C.M.S. Jones, N.S. Bennett, J. Marques-Hueso, Determination of the refractive index of BaY2F8:Er3+ (0.5 mol\% to $30 \mathrm{~mol} \%)$ in the $300 \mathrm{~nm}-1800 \mathrm{~nm}$ range by ellipsometry; a recordbreaking upconversion material, Journal of Luminescence. 230 (2021) 117639.

https://doi.org/10.1016/j.jlumin.2020.117639.

[35] G.E. Arnaoutakis, Novel up-conversion concentrating photovoltaic concepts, Thesis, Heriot-Watt University, 2015. http://www.ros.hw.ac.uk/handle/10399/2933 (accessed August 27, 2016).

[36] M. Rüdiger, S. Fischer, J. Frank, A. Ivaturi, B.S. Richards, K.W. Krämer, M. Hermle, J.C. Goldschmidt, Bifacial n-type silicon solar cells for upconversion applications, Solar Energy Materials and Solar Cells. 128 (2014) 57-68. https://doi.org/10.1016/j.solmat.2014.05.014.

[37] C.A. Gueymard, D. Myers, K. Emery, Proposed reference irradiance spectra for solar energy systems testing, Solar Energy. 73 (2002) 443-467.

[38] A. Riverola, A. Mellor, D. Alonso Alvarez, L. Ferre Llin, I. Guarracino, C.N. Markides, D.J. Paul, D. Chemisana, N. Ekins-Daukes, Mid-infrared emissivity of crystalline silicon solar cells, Solar Energy Materials and Solar Cells. 174 (2018) 607-615. https://doi.org/10.1016/j.solmat.2017.10.002.

[39] A. Richter, J. Benick, F. Feldmann, A. Fell, M. Hermle, S.W. Glunz, n-Type Si solar cells with passivating electron contact: Identifying sources for efficiency limitations by wafer thickness and resistivity variation, Solar Energy Materials and Solar Cells. 173 (2017) 96-105. https://doi.org/10.1016/j.solmat.2017.05.042.

[40] R. Santbergen, R.J.C. van Zolingen, The absorption factor of crystalline silicon PV cells: A numerical and experimental study, Solar Energy Materials and Solar Cells. 92 (2008) 432-444. https://doi.org/10.1016/j.solmat.2007.10.005.

[41] F. Auzel, Spectral narrowing of excitation spectra in n-photons up-conversion processes by energy transfers, Journal of Luminescence. 31-32, Part 2 (1984) 759-761. https://doi.org/10.1016/00222313(84)90116-9.

[42] A. Shalav, B.S. Richards, T. Trupke, K.W. Krämer, H.U. Güdel, Application of NaYF4:Er3+ upconverting phosphors for enhanced near-infrared silicon solar cell response, Appl. Phys. Lett. 86 (2005) 013505. https://doi.org/10.1063/1.1844592.

[43] M. Pollnau, D.R. Gamelin, S.R. Lüthi, H.U. Güdel, M.P. Hehlen, Power dependence of upconversion luminescence in lanthanide and transition-metal-ion systems, Phys. Rev. B. 61 (2000) 3337-3346. https://doi.org/10.1103/PhysRevB.61.3337.

[44] G.E. Arnaoutakis, J.M. Gordon, E.A. Katz, D. Busko, B.S. Richards, Up-conversion threshold under concentrated sunlight, in: 2019 Conference on Lasers and Electro-Optics Europe and European Quantum Electronics Conference (2019), Paper Jsiii_1_4, Optical Society of America, 2019: p. jsiii_1_4. https://www.osapublishing.org/abstract.cfm?uri=CLEO_Europe-2019-jsiii_1_4 (accessed September 28, 2019).

[45] G.E. Arnaoutakis, Dmitry Busko, Bryce S. Richards, Jeffrey Gordon, Eugene A. Katz, Ultrabroadband near-infrared up-conversion for solar energy harvesting, In Preparation. (n.d.).

[46] G.E. Arnaoutakis, J. Marques-Hues, A. Ivaturi, K.W. Krämer, T.K. Mallick, B.S. Richards, Enhancement of upconversion for photovoltaics with $\beta$-NaYF4:Er3+ and concentrating integrated optics, in: Renewable Energy and the Environment (2013), Paper PT3C.4, Optical Society of America, 2013: p. PT3C.4. https://doi.org/10.1364/PV.2013.PT3C.4. 
[47] Q. Liu, H. Liu, D. Li, W. Qiao, G. Chen, H. Ågren, Microlens array enhanced upconversion luminescence at low excitation irradiance, Nanoscale. 11 (2019) 14070-14078. https://doi.org/10.1039/C9NR03105G.

[48] Y. Ji, W. Xu, N. Ding, H. Yang, H. Song, Q. Liu, H. Ågren, J. Widengren, H. Liu, Huge upconversion luminescence enhancement by a cascade optical field modulation strategy facilitating selective multispectral narrow-band near-infrared photodetection, Light Sci Appl. 9 (2020) 184. https://doi.org/10.1038/s41377-020-00418-0.

[49] P. Manley, M. Segantini, D. Ahiboz, M. Hammerschmidt, G. Arnaoutakis, R.W. MacQueen, S. Burger, C. Becker, Double-layer metasurface for enhanced photon up-conversion, APL Photonics. 6 (2021) 036103. https://doi.org/10.1063/5.0040839. 